\title{
Quadratic Equations in a Cyclic Number System
}

\author{
By R. Wilson, University College, Swansea. \\ (Received 8th July 1930. Read 7th November 1930.)
}

\section{§1. Introduction.}

Mr D. E. Littlewood ${ }^{1}$ has recently discussed the properties of the quadratic equation over the real quaternions and shown that the solutions correspond to the common intersections of four quadrics in four-space. Although complex quaternion solutions may arise, the system of real quaternions to which the coefficients belong is a division algebra. It is of interest, therefore, to discuss the solution of the quadratic when the coefficients are drawn from a system containing divisors of zero. The simplest such systems are those defined by $x=x_{0}+x_{1} e_{1}+\ldots+x_{n-1} e_{n-1}$, where the $x$ 's are real and the units form a cyclic group under the product law

$$
e_{r} e_{s}=e_{r+s},(r+s<n) ; e_{r} e_{s}=e_{r+s-n},(r+s \geqslant n) ; e_{0}=1
$$

In this system the commutative law of multiplication holds and the divisors of zero are those numbers for which either

$$
x_{0}+x_{1}+\ldots+x_{n-1}=0 \text {, or } x_{0}=x_{1}=\ldots=x_{n-1},
$$

called for convenience divisors of zero of the first and second kinds. The associated divisor of zero, $y$, such that $x y=0=y x$ is of the opposite kind to $x$. Further the power of a divisor of zero or its product with any number other than its associate is a divisor of zero of the same kind.

The results for the more general number system in which the $x$ 's are complex are quoted in $\S 5$, the reason for treating the more restricted problem in detail being the interest of its geometrical analogue, which involves the intersections of $n$ special quadrics in $n$-space. For brevity, proofs are given for the case $n=3$, the treatment in the general case being similar. Letters $a, b, c, x, \ldots$ without suffixes denote algebraic elements, whose scalar components are indicated by use of suffixes.

\$2. The Leading Coefficient a Non-Divisor of Zero.

The equation will be written $a x^{2}+2 b x+c=0$, where

$$
\begin{array}{ll}
a=a_{1}+a_{2} i+a_{3} j, & c=c_{1}+c_{2} i+c_{3} j, \\
b=b_{1}+b_{2} i+b_{3} j, & x=x_{1}+x_{2} i+x_{3} j,
\end{array}
$$

1 Proc. London Math. Soc. (2), 31 (1930), 40-46. 
and the three units $1, i, j$, of the algebra satisfy the relations

$$
i^{2}=j, \quad j^{2}=i, \quad i j=1=j i \text {. }
$$

The solutions are, by the usual process,

where

$$
x_{1}+x_{2} i+x_{3} j=\frac{-\left(b_{1}+b_{2} i+b_{3} j\right)+\left(u_{1}+u_{2} i+u_{3} j\right)}{a_{1}+a_{2} i+a_{3} j}
$$

$$
\left(u_{1}+u_{2} i+u_{3} j\right)^{2}=\left(b_{1}+b_{2} i+b_{3} j\right)^{2}-\left(a_{1}+a_{2} i+a_{3} j\right)\left(c_{1}+c_{2} i+c_{3} j\right) .
$$

This last gives rise to the equations

$$
\left.\begin{array}{l}
u_{1}^{2}+2 u_{2} u_{3}=\left(b_{1}^{2}-a_{1} c_{1}\right)+\left(2 b_{2} b_{3}-a_{2} c_{3}-a_{3} c_{2}\right), \\
u_{2}^{2}+2 u_{3} u_{1}=\left(b_{2}^{2}-a_{2} c_{2}\right)+\left(2 b_{3} b_{1}-a_{3} c_{1}-a_{1} c_{3}\right), \\
u_{3}^{2}+2 u_{1} u_{2}=\left(b_{3}^{2}-a_{3} c_{3}\right)+\left(2 b_{1} b_{2}-a_{1} c_{2}-a_{2} c_{1}\right),
\end{array}\right\}
$$

whence

$$
\left(u_{1}+u_{2}+u_{3}\right)^{2}=\left(b_{1}+b_{2}+b_{3}\right)^{2}-\left(a_{1}+a_{2}+a_{3}\right)\left(c_{1}+c_{2}+c_{3}\right) .
$$

There are eight solutions to (2) (not necessarily real), except when the discriminant $b^{2}-a c=0$, or when $c$ is a divisor of zero (discussed in $\S 4$ ). In the former case the right hand sides of (2) all become zero, as do $u_{1}, u_{2}, u_{3}$, and the number of solutions reduces to one.

\section{§3. The Leading Coefficient a Divisor of Zero.}

Suppose only $a$ to be a divisor of zero.

The usual method of solution gives $a x=-b+u$, or

$$
\left.\begin{array}{l}
a_{1} x_{1}+a_{3} x_{2}+a_{2} x_{3}=-b_{1}+u_{1}, \\
a_{3} x_{1}+a_{2} x_{2}+a_{1} x_{3}=-b_{2}+u_{2} \\
a_{2} x_{1}+a_{1} x_{2}+a_{3} x_{3}=-b_{3}+u_{3} .
\end{array}\right\}
$$

We shall consider separately the different kinds of divisors of zero.

If $a_{1}+a_{2}+a_{3}=0$, only two of equations (4) are independent, requiring the consistency condition

$$
b_{1}+b_{2}+b_{3}=u_{1}+u_{2}+u_{3},
$$

which is satisfied, as is seen from (3).

To get a third independent equation, multiply the quadratic by $(1+i+j)$, the divisor of zero associated with $a$, giving

$$
(1+i+j)(2 b x+c)=0 \text {. }
$$

It follows that $(2 b x+c)$ must be a divisor of zero of the first kind and hence

$$
2\left(b_{1}+b_{2}+b_{3}\right)\left(x_{1}+x_{2}+x_{3}\right)+\left(c_{1}+c_{2}+c_{3}\right)=0 .
$$

This, with (4), gives a unique value to $x$ for each value of $u$. 
The consistency condition reduces the values of $u$ to four only, which is therefore the number of roots of the quadratic in this case.

If $a_{1}=a_{2}=a_{3}$, only one of equations (4) is independent, requiring the consistency conditions

$$
b_{1}-u_{1}=b_{2}-u_{2}=b_{3}-u_{3}=\lambda,
$$

which are satisfied when $\lambda$ is determined from (2) by

$$
3 \lambda^{2}-2 \lambda\left(b_{1}+b_{2}+b_{3}\right)-a_{1}\left(c_{1}+c_{2}+c_{3}\right)=\mathbf{0} .
$$

Proceeding as above to multiply the quadratic by $\left(C_{1}+C_{2} i+C_{3} j\right)$, where $C_{1}+C_{2}+C_{3}=0$, the $C$ 's being otherwise independent, it follows that $(2 b x+c)$ must have the form $C(1+i+j)$, whence

$$
\begin{aligned}
& \left(b_{1} x_{1}+b_{3} x_{2}+b_{2} x_{3}\right)+c_{1}=\left(b_{3} x_{1}+b_{2} x_{2}+b_{1} x_{3}\right)+c_{2} \\
& =\left(b_{2} x_{1}+b_{1} x_{2}+b_{3} x_{3}\right)+c_{3},
\end{aligned}
$$

giving with (4), three independent linear equations.

The consistency conditions, however, reduce the number of values of $u$, and hence the number of solutions, to two.

The case $a_{1}+a_{2}+a_{3}=0, b_{1}=b_{2}=b_{3}, c_{1}=c_{2}=c_{3}$, gives $x_{1}+x_{2}+x_{3}=0$, while $a_{1}=a_{2}=a_{3}, b_{1}+b_{2}+b_{3}=0, c_{1}+c_{2}+c_{3}=0$ gives $x_{1}=x_{2}=x_{3}$, so that in each case the only solution is $x$ a divisor of zero. (See $\$ 4$.)

\section{§4. Other Cases.}

If $a$ is a divisor of zero, then, as before

$$
2 b x+c=C a \text { or } 2 b x=(C a-c),
$$

where $C$ is real and arbitrary.

If $b$ is also a divisor of zero, then in the same way

$$
c-C a=C^{\prime} b \text { or } c=C a+C^{\prime} b,
$$

where $C, C^{\prime}$, are real and arbitrary.

If $a$ and $b$ are divisors of zero of the same kind, then unless $c$ is a divisor of zero of that kind, there is no solution. On the other hand, if $a$ and $b$ are of opposite kinds there is no restriction on $c$.

We note that if $a, b, c$, are divisors of zero of the first kind, equation (5) vanishes identically and the solutions given by (4) contain an arbitrary constant, while if $a, b, c$, are of the second kind, equations (6) become identities and (4) gives solutions containing two arbitrary constants.

The cases in which the solution is a divisor of zero appear quite simply. For if $x$ is a divisor of zero, then

$$
x(a x+2 b)+c=0,
$$


shows that $c$ must be one also, while if $c$ is a divisor of zero, then either $x$ or $(a x+2 b)$ is one of the same kind. ${ }^{1}$

If $a$ and $c$ are divisors of zero of the same kind and $b$ is not, then $x$ must be, while if $b$ and $c$ are then either $a$ or $x$ is.

The solutions in these cases can be found as in $\$ \S 2$ and 3 .

\section{§5. Extensions.}

The results for the cyclic system with $n$ units can be enunciated from the preceding. If $a$ is not a divisor of zero, there are $2^{n}$ solutions unless the discriminant vanishes when there is only one, or unless $c$ is a divisor of zero. In the latter case $x$ may be a divisor of zero of the same kind and there are $2^{n-1}$ or 2 other solutions according as this is of the first or second kind. If $a$ is a divisor of zero then there are $2^{n-1}$ or 2 solutions according as it is of the first or second kind. If $a, b$ are divisors of zero of the same kind then unless $c$ is also, there is no solution. If $a, b, c$, are divisors of zero of the first kind then the $2^{n-1}$ solutions each involve an arbitrary constant, while if of the second kind the 2 solutions each involve $(n-1)$ arbitrary constants.

When the coefficients $x_{r}$ in the number system are taken over the complex numbers, the problem becomes at once more general and more simple. It is robbed of those eccentricities arising from the coefficients of the quadratic being in a restricted system while the roots belong to the wider system.

The divisors of zero in the new system are those numbers $x_{0}+x_{1} e_{1}+\ldots+x_{n-1} e_{n-1}$ in which $x_{0}+x_{1} \omega+\ldots+x_{n-1} \omega^{n-1}=0$, where $\omega$ is any root of $x^{n}-1=0$. There are thus $2^{n}-2$ divisors of zero, obtained according as one or more of these relations are satisfied by the coefficients of the number. As a result, the following modifications in the number of solutions occur. According as $a$ is a divisor of zero satisfying $1,2, \ldots(n-1)$ relations, there are $2^{n-1}, 2^{n-2}, \ldots$ or 2 solutions, and when $b$ and $c$ are also divisors of zero of the same type there are $1,2, \ldots$ or $(n-1)$ arbitrary constants involved. These constants, as happens also in regard to the results of $\S 4$ for this system, are complex.

1 When $a$ is not a divisor of zero and $c_{1}+c_{2}+c_{3}=0$, then $u_{1}+u_{2}+u_{3}=$ $\pm\left(b_{1}+b_{2}+b_{3}\right)$. From (1) this gives $x$ or $(a x+2 b)$ a divisor of zero of the first kind. Four values of $u$ correspond to each case, giving only four solutions other than $x$ a divisor of zero. On the other hand if $c_{1}=c_{2}=c_{3}$, then for $u_{1}=b_{1}-\lambda, u_{2}=b_{2}-\lambda$, $u_{3}=b_{3}-\lambda$ equations (2) each give $3 \lambda^{2}-2 \lambda\left(b_{1}+b_{2}+b_{3}\right)+c_{1}\left(a_{1}+a_{2}+a_{3}\right)=0$, whence $x_{1}=x_{2}=x_{3}$. Similarly $u_{1}=\lambda-b_{1}, u_{2}=\lambda-b_{2}, u_{3}=\lambda-b_{3}$ gives $(a x+2 b)$ a divisor of zero and produces only two values of $x$. 


\section{§6. Geometrical Aspect-Central Quadrics.}

We shall now give a geometrical analysis of $\$ \S 2-4$ in which $x$ is represented by the point $\left(x_{1}, x_{2}, x_{3}\right)$ in three-space.

Identification of the separate components of the quadratic gives the set of associated quadrics

$$
\left.\begin{array}{l}
a_{1}\left(x_{1}^{2}+2 x_{2} x_{3}\right)+a_{2}\left(x_{2}^{2}+2 x_{3} x_{1}\right)+a_{3}\left(x_{3}^{2}+2 x_{1} x_{2}\right)+2 b_{1} x_{1}+2 b_{3} x_{2}+2 b_{2} x_{3}+c_{1}=0, \\
a_{2}\left(x_{1}^{2}+2 x_{2} x_{3}\right)+a_{3}\left(x_{2}^{2}+2 x_{3} x_{1}\right)+a_{1}\left(x_{3}^{2}+2 x_{1} x_{2}\right)+2 b_{2} x_{1}+2 b_{1} x_{2}+2 b_{3} x_{3}+c_{2}=0, \\
a_{3}\left(x_{1}^{2}+2 x_{2} x_{3}\right)+a_{1}\left(x_{2}^{2}+2 x_{3} x_{1}\right)+a_{2}\left(x_{3}^{2}+2 x_{1} x_{2}\right)+2 b_{3} x_{1}+2 b_{2} x_{2}+2 b_{1} x_{3}+c_{3}=0 .
\end{array}\right\}
$$

The discriminating cubic is the same in each case with the roots $\lambda_{1}=a_{1}+a_{2}+a_{3}, \quad \lambda_{2}, \lambda_{3}= \pm\left(a_{1}^{2}+a_{2}^{2}+a_{3}^{2}-a_{2} a_{3}-a_{3} a_{1}-a_{1} a_{2}\right)^{\frac{1}{2}}$, giving hyperboloids.

If $a$ is not a divisor of zero, none of $\lambda_{1}, \lambda_{2}, \lambda_{3}$ are zero and the quadrics have the common centre satisfying $a_{1} x_{1}+a_{3} x_{2}+a_{2} x_{3}+b_{1}=0, a_{3} x_{1}+a_{2} x_{2}+a_{1} x_{3}+b_{2}=0, a_{2} x_{1}+a_{1} x_{2}+a_{3} x_{3}+b_{3}=0$. Referred to parallel axes through the common centre, the equations of the quadrics take the form

$$
\left.\begin{array}{l}
a_{1}\left(x_{1}^{2}+2 x_{2} x_{3}\right)+a_{2}\left(x_{2}^{2}+2 x_{3} x_{1}\right)+a_{3}\left(x_{3}^{2}+2 x_{1} x_{2}\right)+\left(S_{1} / D\right)=0, \\
a_{2}\left(x_{1}^{2}+2 x_{2} x_{3}\right)+a_{3}\left(x_{2}^{2}+2 x_{3} x_{1}\right)+a_{1}\left(x_{3}^{2}+2 x_{1} x_{2}\right)+\left(S_{2} / D\right)=0, \\
a_{3}\left(x_{1}^{2}+2 x_{2} x_{3}\right)+a_{1}\left(x_{2}^{2}+2 x_{3} x_{1}\right)+a_{2}\left(x_{3}^{2}+2 x_{1} x_{2}\right)+\left(S_{3} / D\right)=0
\end{array}\right\}
$$

where

$$
D=\left|\begin{array}{lll}
a_{1} & a_{3} & a_{2} \\
a_{3} & a_{2} & a_{1} \\
a_{2} & a_{1} & a_{3}
\end{array}\right|, S_{1}=\left|\begin{array}{ccc}
U_{1} & U_{2} & U_{3} \\
a_{3} & a_{2} & a_{1} \\
a_{2} & a_{1} & a_{3}
\end{array}\right|, S_{2}=\left|\begin{array}{ccc}
U_{2} & U_{3} & U_{1} \\
a_{3} & a_{2} & a_{1} \\
a_{2} & a_{1} & a_{3}
\end{array}\right|, S_{3}=\left|\begin{array}{ccc}
U_{3} & U_{1} & U_{2} \\
a_{3} & a_{2} & a_{1} \\
a_{2} & a_{1} & a_{3}
\end{array}\right|,
$$

$U_{1}, U_{2}, U_{3}$, being the right hand sides of (2), in order.

The principal directions of the three quadrics are given by

$$
\left.\begin{array}{l}
a_{i} l_{r}+a_{j} m_{r}+a_{k} n_{r}=\lambda_{r} l_{r} \\
a_{j} l_{r}+a_{k} m_{r}+a_{i} n_{r}=\lambda_{r} m_{r} \\
a_{k} l_{r}+a_{i} m_{r}+a_{j} n_{r}=\lambda_{r} n_{r}
\end{array}\right\}
$$

where

$$
\begin{array}{rlrl}
(i, j, k) & =(1,3,2) \text { for } r & =1 \\
& =(2,1,3) & r & =2 \\
& =(3,2,1) & r & =3 .
\end{array}
$$

It will be seen that by the cyclic interchange of $(l, m, n)$ the three sets of relations permute into one another. If $O X, O Y, O Z$, are the coordinate axes of (9), it follows that the principal axes of the three quadrics have the same orientation with respect to the trihedrals $O X Y Z, O Y Z X, O Z X Y$, respectively. If the direction cosines of the principal axes of the first quadric are

$$
\left(l_{1}, m_{1}, n_{1}\right), \quad\left(l_{2}, m_{2}, n_{2}\right), \quad\left(l_{3}, m_{3}, n_{3}\right) \text {, }
$$


the angle between a corresponding pair from the second and third quadrics is $\cos ^{-1}\left(m_{r} n_{r}+n_{r} l_{r}+l_{r} m_{r}\right)$. From (10) we get

$$
\left(l_{r}+m_{r}+n_{r}\right)\left(a_{1}+a_{2}+a_{3}-3 \lambda\right)=0 \text {, or } l_{r}+m_{r}+n_{r}=0 .
$$

Consequently $m_{r} n_{r}+n_{r} l_{r}+l_{r} m_{r}=-\frac{1}{2}$, and the angle is $120^{\circ}$ in the case of every corresponding pair of axes chosen from any pair of the quadrics.

Hence the principal axes of the three quadrics pass into one another on rotation through $120^{\circ}$ about the line $x_{1}=x_{2}=x_{3}$.

The quadrics clearly intersect in eight points real or imaginary, as is seen by solving (9) for $x_{1}^{2}+2 x_{2} x_{3}, x_{2}^{2}+2 x_{3} x_{1}, x_{3}^{2}+2 x_{1} x_{2}$.

That they are similar quadrics appears from the canonical forms

$$
\lambda_{1} x^{2}+\lambda_{2} y^{2}+\lambda_{3} z^{2}+\left(S_{r} / D\right)=0,(r=1,2,3) .
$$

The condition that the discriminant of the quadratic should vanish is, from (2), $U_{1}=U_{2}=U_{3}=0$, giving from (9) and (12), $S_{1}=S_{2}=S_{3}=0$, so that in this case the quadrics become equal cones symmetrically set with a common vertex at their only point of intersection.

If $c_{1}+c_{2}+c_{3}=0$ the common intersections of the quadrics lie in fours in the planes

$$
x_{1}+x_{2}+x_{3}=0,\left(a_{1}+a_{2}+a_{3}\right)\left(x_{1}+x_{2}+x_{3}\right)+2\left(b_{1}+b_{2}+b_{3}\right)=0 .
$$

If $c_{1}=c_{2}=c_{3}$, the quadrics are identical except for position and touch in the four points common to the quadrics and the lines

$$
\begin{aligned}
x_{1}=x_{2}=x_{3},\left(a_{1} x_{1}+a_{3} x_{2}+a_{2} x_{3}\right)+2 b_{1} & =\left(a_{2} x_{1}+a_{1} x_{2}+a_{3} x_{3}\right)+2 b_{2} \\
& =\left(a_{3} x_{1}+a_{2} x_{2}+a_{1} x_{3}\right)+2 b_{3} .
\end{aligned}
$$

§7. The Paraboloids.

If $a_{1}+a_{2}+a_{3}=0$, then $\lambda_{1}=0$, while $\lambda_{2}, \lambda_{3} \neq 0$. Hence the three quadrics become hyperbolic paraboloids having the principal direction corresponding to $\lambda_{1}=0,(1: 1: 1)$ in common. As with the central quadrics the other corresponding principal directions are set at $120^{\circ}$. In the case of the direction corresponding to $\lambda_{1}=0$, this is not true (as with the central quadrics) for $l_{1}+m_{1}+n_{1}=\sqrt{3}$ and (11) is satisfied in virtue of $\left(a_{1}+a_{2}+a_{3}-3 \lambda_{1}\right)=0$.

Hence the axes of the three paraboloids are parallel but not coincident and the surfaces thus intersect in four points, real or imaginary.

That they are similar follows from the canonical forms

$$
\lambda_{2}\left(x_{1}^{2}-x_{2}^{2}\right)+2 \sqrt{S_{r}} x_{3}=0,(r=1,2,3) .
$$


They have a common vertex only when the six equations

$$
\begin{aligned}
& 3\left(a_{1} x_{1}+a_{3} x_{2}+a_{2} x_{3}\right)+\left(2 b_{1}-b_{3}-b_{2}\right)=0, \\
& 3\left(a_{3} x_{1}+a_{2} x_{2}+a_{1} x_{3}\right)+\left(2 b_{2}-b_{1}-b_{3}\right)=0, \\
& 3\left(a_{2} x_{1}+a_{1} x_{2}+a_{3} x_{3}\right)+\left(2 b_{3}-b_{2}-b_{1}\right)=0, \\
& \left(x_{1}+x_{2}+x_{3}\right)\left(b_{1}+b_{2}+b_{3}\right)+3\left(b_{1} x_{1}+b_{3} x_{2}+b_{2} x_{3}+c_{1}\right)=0, \\
& \left(x_{1}+x_{2}+x_{3}\right)\left(b_{1}+b_{2}+b_{3}\right)+3\left(b_{2} x_{1}+b_{1} x_{2}+b_{3} x_{3}+c_{2}\right)=0, \\
& \left(x_{1}+x_{2}+x_{3}\right)\left(b_{1}+b_{2}+b_{3}\right)+\mathbf{3}\left(b_{3} x_{1}+b_{2} x_{2}+b_{1} x_{3}+c_{3}\right)=0,
\end{aligned}
$$

are satisfied simultaneously.

For this it is necessary and sufficient that $b_{1}+b_{2}+b_{3}=0$, $c_{1}+c_{2}+c_{3}=0$, in addition to $a_{1}+a_{2}+a_{3}=0$. In this case the $S_{r}$ are all zero as is also $D$, and we get three pairs of planes, each of canonical form $x_{1}^{2}-x_{2}^{2}=0$, having a common line of intersection parallel to $x_{1}=x_{2}=x_{3}$. The bisectors of each pair of planes are inclined to one another at $120^{\circ}$.

If $a_{1}+a_{2}+a_{3}=0, b_{1}=b_{2}=b_{3}, c_{1}=c_{2}=c_{3}$, then the paraboloids have $x_{1}=x_{2}=x_{3}$ as a common generator.

\section{§8. The Cylinders.}

If $a_{1}=a_{2}=a_{3}$ the surfaces (7) become

$$
a_{1}\left(x_{1}+x_{2}+x_{3}\right)^{2}+2 b_{1} x_{1}+2 b_{3} x_{2}+2 b_{2} x_{3}+c_{1}=0, \text { etc., }
$$

or, in canonical form,

$a_{1}\left(x_{1}+x_{2}+x_{3}+k\right)^{2}=2 x_{1}\left(a_{1} k-b_{1}\right)+2 x_{2}\left(a_{1} k-b_{3}\right)+2 x_{3}\left(a_{1} k-b_{2}\right)+\left(a_{1} k^{2}-c_{1}\right)$, where

$$
3 a_{1} k=b_{1}+b_{2}+b_{3} .
$$

These are parabolic cylinders having the common axial plane

$$
3 a_{1}\left(x_{1}+x_{2}+x_{3}\right)+\left(b_{1}+b_{2}+b_{3}\right)=0,
$$

and having the tangent planes at the vertex generators intersecting at $120^{\circ}$, these last meeting in a common line only if $3 a_{1} k^{2}=c_{1}+c_{2}+c_{3}$. The surfaces have only two points in common.

If further $b_{1}=b_{2}=b_{3}$ then the surfaces degenerate into parallel sets of parallel planes. These coincide in one plane only if $c_{1}=c_{2}=c_{3}$.

If $a_{1}=a_{2}=a_{3}, b_{1}+b_{2}+b_{3}=0, c_{1}+c_{2}+c_{3}=0$, the points of intersection of the cylinders lie in the plane $x_{1}+x_{2}+x_{3}=0$, and the axial planes have, as above, a common line of intersection. 